\title{
Short-term Effect of Ambient Air Pollutants Change on the Risk of Tuberculosis Outpatient Visits: A Time-series Study in Fuyang, China
}

\section{Xin-Qiang Wang}

Anhui Medical University School of Public Health

\section{Ying-Qing Li}

Anhui Medical University School of Public Health

Cheng-Yang Hu

Anhui Medical University School of Public Health

Kai Huang

Anhui Medical University School of Public Health

Kun Ding

Anhui Medical University School of Public Health

Xiao-Jing Yang

Anhui Medical University School of Public Health

Xin Cheng

Anhui Medical University School of Public Health

Kang-Di Zhang

Anhui Medical University School of Public Health

Wen-Jie Yu

Anhui Medical University School of Public Health

Jie Wang

Anhui Medical University School of Public Health

\section{Yong-Zhong Zhang}

Anhui Chest Hospital

\section{Zhen-tao Ding}

Fuyang Center for Disease Control and Prevention

\section{Xiu-Jun Zhang}

Anhui Medical University School of Public Health

Xiao-Hong Kan ( $\nabla$ ahfidelis@126.com )

Anhui Chest Hospital https://orcid.org/0000-0002-0903-6937 
Keywords: Tuberculosis, Risk of outpatient visit, Ambient air pollutants, DLNM, Time-series study, China

Posted Date: August 24th, 2021

DOI: https://doi.org/10.21203/rs.3.rs-789004/v1

License: (c) (1) This work is licensed under a Creative Commons Attribution 4.0 International License. Read Full License

Version of Record: A version of this preprint was published at Environmental Science and Pollution Research on January 7th, 2022. See the published version at https://doi.org/10.1007/s11356-021-173237. 
2 Title: Short-term effect of ambient air pollutants change on the risk of tuberculosis

3 outpatient visits: A time-series study in Fuyang, China

4 Authors: Xin-Qiang Wang ${ }^{\mathrm{a}, 1}$, Ying-Qing $\mathrm{Li}^{\mathrm{a}, 1}$, Cheng-Yang $\mathrm{Hu}^{\mathrm{d}}$, Kai Huang ${ }^{\mathrm{a}}$, Kun

$5 \quad$ Ding $^{\mathrm{a}}$, Xiao-Jing Yang ${ }^{\mathrm{a}}$, Xin Cheng ${ }^{\mathrm{a}}$, Kang-Di Zhang ${ }^{\mathrm{a}}$, Wen-Jie Yu ${ }^{\mathrm{a}}$, Jie Wang ${ }^{\mathrm{a}}$, Yong-

6 Zhong Zhang ${ }^{\mathrm{e}}$, Zhen-tao Ding ${ }^{\mathrm{f}}$, Xiu-Jun Zhang ${ }^{\mathrm{a} * *}$, Xiao-Hong Kan ${ }^{\text {b,c* } *}$

\section{Affiliations:}

8 a: Department of Epidemiology and Biostatistics, School of Public Health, Anhui

9 Medical University, 81 Meishan Road, Hefei, 230032, China;

b: Anhui Medical University Clinical College of Chest, 397 Jixi Road, Hefei, 230022,

11 China;

c: Anhui Chest Hospital, 397 Jixi Road, Hefei, 230022, China;

d: Department of Humanistic Medicine, School of Humanistic Medicine, Anhui

Medical University, 81 Meishan Road, Hefei, 230032, China;

e: Anhui Institute of Tuberculosis Prevention and Control, 397 Jixi Road, Hefei, 230022,

China;

f: Fuyang Provincial Center for Disease Control and Prevention, 19 Zhongnan Avenue, Fuyang, 236030, China;

\section{*Corresponding authors:}

1. Xiao-Hong Kan, Anhui Medical University Clinical College of Chest, 397 Jixi Road,

21 Hefei, 230022, China;

$22 \quad$ Tel: +055163615304 
2. Xiu-Jun Zhang, PhD, Department of Epidemiology and Biostatistics, School of Public Health, Anhui Medical University, 81 Meishan Road, Hefei, 230032, China; Tel: + 865515167743 ;

E-mail: zhangxiujun@ahmu.edu.cn

ORCID: 0000-0002-8123-185X

\section{Running title: Association between ambient air pollutants and TB}

Xin-Qiang Wang and Ying-Qing Li contributed equally to this article.

\section{Abstract:}

There is growing evidence that air pollution plays a role in $\mathrm{TB}$, but few studies have comprehensively included the six common air pollutants. Our objectives were to investigate the association between short-term exposure to six common air pollutants and the risk of tuberculosis outpatient visits in Fuyang, China, during the period 20152020. We combined the two models to explore the effects of exposure to six air pollutants on the risk of tuberculosis outpatient visits, including Poisson generalized linear regression model and distributed lag non-linear model (DLNM). We performed stratified analyses for season, gender and age. We used the lag-specific relative risks and cumulative relative risk obtained by increasing pollutant concentration by per 10 units to evaluate the connection between six air pollutants and $\mathrm{TB}, \mathrm{PM}_{2.5}(\mathrm{RR}=1.0018$, 95\%CI: 1.0004-1.0032, delay of 12 days) and (RR=1.0169,95\%CI: $1.0007-1.0333$, lag 
0-16 days), $\mathrm{SO}_{2}$ were 0.9549 (95\%CI: 0.9389-0.9712, lag 0 days) and 0.8212 (95\%CI: 0.7351-0.9173, 0-20 days lag). Stratified analyses showed that seasonal differences had a greater impact on TB, and male more likely to develop TB than female, older people more likely to develop TB than younger people. Exposure to $\mathrm{O}_{3}, \mathrm{CO}, \mathrm{PM}_{10}, \mathrm{PM}_{2.5}$, and $\mathrm{NO}_{2}$ increases the risk of $\mathrm{TB}$ outpatient visits, except $\mathrm{SO}_{2}$ reduces the risk. Male and elderly people should be protected. We need to pay more attention to the impact of seasonal effects on TB.

Keywords: Tuberculosis; Risk of outpatient visit; Ambient air pollutants; DLNM; Time-series study; China.

\section{Introduction}

Tuberculosis (TB) is a chronic contagion caused by tuberculosis bacilli infection. Mycobacterium TB may invade all sorts of organs of human body, but basically encroach on lung, call pulmonary tuberculosis (PTB). Tuberculosis is a global public health problem that seriously endangers public health. Tuberculosis is one of the most dangerous infectious diseases, causing the world's top 10 deaths (Wang and Guo et al., 2021). There are about 10 million new cases and 1.5 million deaths caused by TB every year. China is a country with a high burden of tuberculosis, ranking second in the world. It is reported that in 2019, an estimated 8.9 million to 11 million people worldwide were infected with TB, with China ranking third, accounting for 8.4 percent of the global total (WHO, 2020). We know from previous studies that poor nutrition, weather, 
diabetes, smoking, weakened immune systems, HIV/AIDS, air pollution, alcohol consumption and many other factors all play a large or small role in the progression of TB (Kim, 2014; Rivas-Santiago and Sarkar et al., 2015; Elf and Eke et al., 2017; Li and Mao et al., 2019).

With the development and deepening of epidemiological studies, more and more evidences show the influence of air pollutants on tuberculosis. A recent review summarized the links and potential mechanisms between respiratory bacterial infections and air pollution, described the association between various air pollutants and the occurrence and development of tuberculosis by analyzing existing epidemiological studies (Pompilio and Di Bonaventura, 2020). Another literature review also showed that the pollutant with the greatest impact on TB was fine particulate matter $\mathrm{PM}_{2.5}(<2.5 \mu \mathrm{m})$, nitrogen dioxide $\left(\mathrm{NO}_{2}\right)$, fine particulate matter $\mathrm{PM}_{10}(<10 \mu \mathrm{m})$ and sulfur dioxide $\left(\mathrm{SO}_{2}\right)$ also showed significant correlation (Popovic and Soares Magalhaes et al., 2019). A meta-analysis published in March of this year pooled 17 related studies and suggested that long-term exposure to $\mathrm{PM}_{10}, \mathrm{SO}_{2}$ or $\mathrm{NO}_{2}$ was associated with higher TB incidence (Xiang and Xu et al., 2021). An ecological study conducted in the United States, China, and Canada has observed a correlation between coal consumption and tuberculosis. The decline in TB rates has coincided with a decline in household coal consumption, indicating the link between air pollution and TB (Tremblay, 2007). However, the available results on the relationship between air pollution and TB risk are inconsistent. Two studies in Mexico have shown that exposure to airborne particle inhibits the body's immune response to the TB bacterium. $\mathrm{PM}_{2.5}$ and 
$\mathrm{PM}_{10}$ can inhibit the human body to produce cytokines and antimicrobial peptides, impair the immunity of the human lung, promotes the occurrence and development of TB (Sarkar and Rivas-Santiago et al., 2019; Torres and Carranza et al., 2019). However, other studies indicated that short-term or long-term exposure to $\mathrm{PM}_{2.5}$ or $\mathrm{PM}_{10}$ was not associated with TB risk (Liu and Cui et al., 2018; Xu and Liao et al., 2019; Xiang and $\mathrm{Xu}$ et al., 2021). A study in Seoul, South Korea, has confirmed that the interquartile increase in $\mathrm{SO}_{2}$ concentration was associated with a $7 \%$ increment in $\mathrm{TB}$ incidence (Hwang and Kang et al., 2014), but a recent study conducted in Hefei, suggests that exposure to $\mathrm{SO}_{2}$ is inversely associated with tuberculosis (Huang and Ding et al., 2020). Exposure to pollutants such as $\mathrm{NO}_{2}$ and ozone $\left(\mathrm{O}_{3}\right)$ can affect the number of TB clinics (Lin and Lin et al., 2019; Huang and Ding et al., 2020; Liu and Zhao et al., 2021). In addition, although there is a lot of research in developed countries, but, still less research in China, so more research is urgently needed to assess the relationship between TB and air pollution in China. Fuyang located in the northwest of Anhui Province, is a city with a large population. Because of the rapid development of economy and the constant growth of population, Fuyang is facing a serious air pollution problem.

The association between various air pollutants and TB has also been explored in time series studies conducted in multiple Chinese cities (Chengdu, Hefei and Wuhan) (Zhu and Xia et al., 2018; Huang and Ding et al., 2020; Huang and Xiang et al., 2020). However, few studies have comprehensively explored the effects of exposure to six pollutants. Therefore, we aim to explore the relationship between daily exposure to 
111

112

113

114

115

116

117

118

119

120

121

122

123

124

125

126

$\mathrm{PM}_{10}$ (particulate matter $<10 \mu \mathrm{m}$ in aerodynamic diameter), sulfur dioxide $\left(\mathrm{SO}_{2}\right), \mathrm{PM}_{2.5}$ (particulate matter $<2.5 \mu \mathrm{m}$ in aerodynamic diameter), nitrogen dioxide $\left(\mathrm{NO}_{2}\right)$, ozone $\left(\mathrm{O}_{3}\right)$, carbon monoxide $(\mathrm{CO})$ and the risk of tuberculosis outpatient visits in Fuyang. Subgroup analyses for seasonal variation, sex, and age were also performed to assess their correction effects.

\section{Material and methods}

\subsection{Ethical statement}

This study was approved by the Anhui Medical University Ethics Committee. All patient information included in the study was unidentified and anonymous.

\subsection{Study location}

Fuyang $\left(114^{\circ} 52^{\prime}-116^{\circ} 49^{\prime} \mathrm{E}, 32^{\circ} 25^{\prime}-34^{\circ} 04^{\prime} \mathrm{N}\right)$ is located in the southern part of the Huang-Huai-Hai Plain. It is a northwest city with the largest population in Anhui Province (Fig. 1). By the end of 2019, the permanent resident population of Fuyang City was 8.259 million, an increase of 52000 over the previous year, with a year-onyear increase of $0.63 \%$. Fuyang city is located in the southern margin of the warm temperate zone, a warm temperate semi-humid monsoon climate. The four seasons are distinct and the rainfall is moderate. Because Fuyang is near the Huaihe River in the 
south, and the north subtropical humid monsoon climate is in the south of the Huaihe River, the climate of Fuyang is characterized by a transition zone from warm temperate zone to north subtropical zone (Anhui Provincial Bureau of Statistics of China, 2020). In recent years, due to population growth and economic development, Fuyang City has faced a serious air pollution problem.

\subsection{Tuberculosis data}

Tuberculosis is a Class B infectious disease, which is one of the infectious diseases that must be reported in China. All case reports in this study were collected from the Tuberculosis Prevention and Control Institute of Anhui Chest Hospital and reported by Fuyang Center for Disease Control and Prevention. We collected admission information for all TB patients from January 1, 2015 to December 31, 2020, including age, gender, home address and date of current visit. In addition, because of the long incubation period of TB, typically between four and eight weeks, the patient's time of infection is hard to confirm. Therefore, we included both recurrent and new cases in this study (Huang and Yang et al., 2020).

\subsection{Environmental data (including meteorological data and pollution data)}

The meteorological data and pollutant data in this study are from China Meteorological Data Network and Fuyang Environmental Monitoring Center 
respectively (http://data.cma.cn/). Pollutants include $\mathrm{PM}_{2.5}, \mathrm{PM}_{10}, \mathrm{SO}_{2}, \mathrm{NO}_{2}\left(\mu \mathrm{g} / \mathrm{m}^{3}\right.$, 24h), $\mathrm{O}_{3}\left(\mu \mathrm{g} / \mathrm{m}^{3}, 8 \mathrm{~h}\right)$ and $\mathrm{CO}\left(\mathrm{mg} / \mathrm{m}^{3}, 24 \mathrm{~h}\right)$. Data were collected from eight fixed monitoring stations in Fuyang. The daily meteorological data we collect are relative humidity $(\mathrm{RH}, \%)$ and mean temperature $\left(\mathrm{MT},{ }^{\circ} \mathrm{C}\right)$.

\subsection{Statistical analysis}

\subsubsection{Descriptive statistical}

The demographic characteristics of tuberculosis were analyzed using simple descriptive statistical methods, including sex, age, etc. A simple descriptive analysis of the distribution of air pollutant data and meteorological data was also carried out. The correlation among meteorological factors, air pollutants and tuberculosis outpatient visits was described by Spearman correlation coefficient and scatter plot.

\subsubsection{Construction of statistical model}

Based on previous biological findings that ambient air pollutants have a delayed effect on health outcomes, most epidemiological studies have found nonlinear exposure-response relationships (Chen and Deng et al., 2017; Guo and Ma et al., 2017). So, we used a distributed lag nonlinear model (DLNM) to examine the relationship between air pollutants and the risk of tuberculosis outpatient visits (Gasparrini, 2014). 
In addition, because the numbers of daily reports of TB outpatients were generally considered to be rare events, we used a generalized linear model (GLM) based on the quasi-Poisson distribution to construct a function fitting the exposure-response and effects of lag (Huang and Ding et al., 2020). First, in order to understand the influence of exposure to six air pollutants in the risk of tuberculosis outpatient visits, we used the function of natural cubic spline ( 7 degrees of freedom per year) to control for long-term trends and seasonal variations (Bhaskaran and Gasparrini et al., 2013; Zhu and Xia et al., 2018). Meanwhile, the ns function with 3 degrees of freedom is used to control the mean temperature (MT) and relative humidity $(\mathrm{RH})$ of meteorological factors (Chen and Chu et al., 2010; Chen and Deng et al., 2017). We also controlled for the day of the week (Dow) and Holiday effect.

Then, we inquired two-dimensional connection between air pollutants and the exposure, lag and response of tuberculosis outpatient visits by the cross-basis functions, with the initial maximum lag day set at 28 days (TB incubation period 28-56 days), using linear cubic spline function fitting exposed - response relationship, natural cubic spline function fitting lag-response relationship, because the purpose of this study was to investigate the effects of short-term exposure. Therefore, we calculated different cumulative lag effects of different pollutants through a single pollutant model, selected the lag days when the first peak or lowest value was reached, and obtained the QAIC (quasi-Poisson Akaike information criteria) values of different lag days (Supplementary Table S1). Finally, the best lag date was selected by QAIC (Gasparrini and Armstrong et al., 2010; Huang and Ding et al., 2020). In order to solve the collinearity relationship 
between air pollutants and meteorological factors, we only added variables with correlation values less than 0.7 into the model (Liu and Tian et al., 2017). The basic model was as follows:

At $\sim$ quasiPoisson (et)

$\log (e t)=\alpha+W_{X}^{T} \eta+n s\left(\right.$ time, $7^{*}$ year $)+n s\left(M T, d f_{1}\right)+n s\left(R H, d f_{2}\right)$

$$
+\left(\beta^{*} D O W\right)+\left(\gamma^{*} \text { Hoilday }\right)
$$

At is the actual tuberculosis outpatient visits in $t$ days, et is the expected tuberculosis outpatient visits t days, $W_{X}^{T} \eta$ is a cross- basis function, $n s$ represents the natural cubic spline function, $d f_{1}$ and $d f_{2}$ represent the degrees of freedom of average temperature and relative humidity respectively, $\beta$ and $\gamma$ are the regression coefficients of Dow and Holiday respectively.

We regarded the Level 1 concentration limits as a reference in the National Environmental Quality Standard (GB3059-2012), used RR (relative risk) estimates and 95\% CI (confidence intervals) to represent the lag-specificity and cumulative risk of TB outpatient visits with each 10-unit increasing in six air pollutants concentrations. After a single-pollutant regression model, we modeled the effects of air pollution by gender (male female), age ( $<15$ years old 15-65 years old $>65$ years old) and season (cold warm). Calculate the RR and 95\% CI using the following formula:

$$
\left(\widehat{E_{1}}-\widehat{E_{2}}\right) \pm 1.96 \sqrt{\left(\widehat{S E}_{1}\right)^{2}+\left(\widehat{S E}_{2}\right)^{2}}
$$

Where $\widehat{E_{1}}$ and $\widehat{E_{2}}$ are the point estimates for the two subgroups, and $\widehat{S E}_{1}$ and $\widehat{S E}_{2}$ are the corresponding standard errors, respectively (Huang and Ding et al., 2020). To test the robustness of the results, we also performed sensitivity analysis: (a) the 
multi-pollutant models were used to adjust for confounding factors among air pollutants;

(b) different degrees of freedom were taken in the ns function of time variables (4-6 df);

(c) changing the degrees of freedom in the ns function of meteorological variables (4$7 \mathrm{df}$ ). All analyses were performed through the "splines", "dlnm" and "mgcv" packages in the R software (version 4.0.0).

\section{Results}

\subsection{Descriptive results}

Table1 provides summary statistics of the variables in this study, include TB case, atmospheric pollutants and meteorology measure. The daily mean concentration of $\mathrm{PM}_{2.5}$ was $55.18 \mu \mathrm{g} / \mathrm{m}^{3}\left(0-276 \mu \mathrm{g} / \mathrm{m}^{3}\right), \mathrm{PM}_{10}$ was $85.89 \mu \mathrm{g} / \mathrm{m}^{3}\left(0-379 \mu \mathrm{g} / \mathrm{m}^{3}\right), \mathrm{SO}_{2}$ was $13.33 \mu \mathrm{g} / \mathrm{m}^{3}\left(2-237 \mu \mathrm{g} / \mathrm{m}^{3}\right), \mathrm{CO}$ was $0.77 \mathrm{mg} / \mathrm{m}^{3}\left(2-2.9 \mathrm{mg} / \mathrm{m}^{3}\right), \mathrm{NO}_{2}$ was $32.09 \mu \mathrm{g} / \mathrm{m}^{3}$ (9 - $\left.93 \mu \mathrm{g} / \mathrm{m}^{3}\right), \mathrm{O}_{3}$ was $92.77 \mu \mathrm{g} / \mathrm{m}^{3}\left(0-226 \mu \mathrm{g} / \mathrm{m}^{3}\right)$. From 2015 to 2020, there were 21,591 cases of tuberculosis, 6384 females and 15,207 males. Among them, 0.94\% were younger than 15 years old, $30.26 \%$ were older than 65 years old, and $68.8 \%$ were between 15 and 65 years old. The minimum and maximum daily mean temperature were $7.8^{\circ} \mathrm{C}$ and $33.5^{\circ} \mathrm{C}$ respectively. The minimum value of relative humidity is $33 \%$ and the maximum is $99 \%$. Fig. 2 shows Spearman correlation coefficient and scatter plot. The distribution of air pollutant concentrations and tuberculosis hospitalizations from 2015 to 2020 are presented in Fig. 3. 
3.2 Relations analysis

3.2.1 Overall association between daily TB outpatient visits and air pollutants

As shown in Supplementary Fig. S1, from the overall exposure response effect, it can be concluded that the exposure concentrations of $\mathrm{PM}_{10}, \mathrm{PM}_{2.5}, \mathrm{NO}_{2}, \mathrm{O}_{3}$ and $\mathrm{CO}$ have a positive relationship with the risk of tuberculosis, while $\mathrm{SO}_{2}$ has a negative relationship with the risk of tuberculosis.

3.2.2 Association between daily TB outpatient visits and $P M_{2.5}$

We can see from the Fig. 4, when a $10 \mu \mathrm{g} / \mathrm{m}^{3}$ increase in $\mathrm{PM}_{2.5}$ concentration, the risk of $\mathrm{TB}$ increased, and the association persisted over a 12 to 20 day delay $(\mathrm{RR}=1.0018,95 \% \mathrm{CI}: 1.0004-1.0032$, delay of 12 days; $\mathrm{RR}=1.0019,95 \% \mathrm{CI}: 1.0004-$ 1.0035 , delay of 20 days). At the same time, the cumulative lag effect reached statistical significance in $0-16$ days $(\mathrm{RR}=1.0169,95 \% \mathrm{CI}: 1.0007-1.0333$, lag $0-16$ days). Subgroup analysis showed that the effects of $\mathrm{PM}_{2.5}$ exposure in male, 15 to 65 years old, and $\geq 65$ years old subgroup were statistically significant. However, an opposite result was found in the cold and warm subgroup, cold ( $\mathrm{RR}=1.0025,95 \% \mathrm{CI}$ : 1.0002 1.0047, lag 13 days) and warm ( $\mathrm{RR}=0.9947,95 \% \mathrm{CI}$ : 0.9899-0.9994, lag 2 days) (Fig.

5, Fig. 6 and Supplementary Table S2). 
3.2.3 Association between daily TB outpatient visits and PM 10

In the single pollutant model, the daily lag effect of increasing $\mathrm{PM}_{10}$ concentration

by $10 \mu \mathrm{g} / \mathrm{m}^{3}$ reached statistical significance on the 14 th day, and lasted until the 20 th

day $(\mathrm{RR}=1.001,95 \% \mathrm{CI}: 1.0002-1.0019$, with a 14 -day lag. $\mathrm{RR}=1.0013,95 \% \mathrm{CI}$ :

1.0002-1.0024, lag 20 days). The cumulative lag effect reached statistical significance

when the lag was $0-21$ days $(\mathrm{RR}=1.0137,95 \% \mathrm{CI}$ : $1.0006-1.027$, lag $0-21$ days $)$. The

stratified analysis showed that the effects of $\mathrm{PM}_{10}$ exposure were statistically significant

in male, 15-60 years old, and cold subgroups. But similar to $\mathrm{PM}_{2.5}$, there was an

opposite effect in the warm season subgroup $(\mathrm{RR}=0.996,95 \% \mathrm{CI}$ : 0.9926-0.9994, with

a lag of 2 days) (Fig. 4, Fig. 5, Fig. 6 and Supplementary Table S3).

\subsubsection{Association between daily TB outpatient visits and CO}

CO single pollutant model reached statistical significance $(R R=1.0037$,

95\%CI :1.0005-1.0068, lag 11 days). The cumulative lag effect of CO exposure showed

a bimodal distribution. The first time was statistically significant with a lag of 0-1 days

When stratified by sex, age, and season, the effect of $\mathrm{CO}$ exposure was still statistically 
significant in male, 15-65 years old, > 65 years old, and cold quarter subgroups (Fig. 5, Fig. 6 and Supplementary Table S4).

\subsubsection{Association between daily TB outpatient visits and $\mathrm{O}_{3}$}

Fig. 4 shows that $\mathrm{O}_{3}$ exposure immediately increased the risk of $\mathrm{TB}$ outpatient visits $(\mathrm{RR}=1.049,95 \% \mathrm{CI}: 1.0012-1.0086,0$ days lag). The cumulative risk curve was bimodal and statistically significant after 0-4 and 0-9 days of lag. In the subgroup analysis, the effect of $\mathrm{O}_{3}$ had a short-term significant effect in male, female, $>65$ years old, 15-65 years old, and cold quarter subgroup (Fig. 5, Fig. 6 and Supplementary Table S5).

3.2.6 Association between daily TB outpatient visits and $\mathrm{SO}_{2}$

Different from other pollutants, when an increase of $10 \mu \mathrm{g} / \mathrm{m}^{3}$ in $\mathrm{SO}_{2}$ concentration, the risk of TB outpatient visits was reduced. In the single pollutant model, the singleday lag risk of $\mathrm{SO}_{2}$ reached statistical significance at 0 days $(\mathrm{RR}=0.9549,95 \% \mathrm{CI}$ : 0.9389-0.9712, lag 0 days). The cumulative lag risk reached its lowest at 20 days (RR $=0.8212,95 \% \mathrm{CI}: 0.7351-0.9173,0-20$ days lag). In the subgroup analysis, all subgroups except the $<15$ years old had statistical significance (Fig. 5, Fig. 6 and Supplementary Table S6). 
As shown in the Fig. 4, the cumulative risk reached statistical significance with a lag of 0-2 days $(\mathrm{RR}=1.0242,95 \% \mathrm{CI}: 1.0059-1.0428$, lag $0-2$ days $)$. Stratification results showed that $\mathrm{NO}_{2}$ exposure was still significant in the cold season group $(\mathrm{RR}=1.0329,95 \% \mathrm{CI}$ : 1.0114-1.0548, lag 0 days) (Fig. 5, Fig. 6 and Supplementary Table S7). the time variable ns (4-6df) and the meteorological variable ns (4-7df), the main results showed no significant change (Table 2 and Supplementary Table S8-25). The results show that the model has good performance and the calculation results are reliable.

\section{Discussion} outpatient visits, and $\mathrm{SO}_{2}$ reduces the risk. In addition, subgroup analysis showed that the pollutants had a greater impact on men and the elderly. The multi-pollutant model 
results showed that the effects of $\mathrm{PM}_{2.5}, \mathrm{CO}, \mathrm{SO}_{2}$ and $\mathrm{NO}_{2}$ on the $\mathrm{TB}$ visit risk remained stable after adjustment, while the effects on $\mathrm{PM}_{10}$ and $\mathrm{O}_{3}$ were reduced to some degree. The findings of this study, which are supported by several previous studies, suggest that tuberculosis is affected by $\mathrm{PM}_{2.5}$ concentrations (Jassal and Bakman et al., 2013; Smith and Schoenbach et al., 2014; Liu and Tian et al., 2017). Two recent time series studies in the Chinese cities of Jinan and Hefei also confirmed the risk of $\mathrm{PM}_{2.5}$ on tuberculosis (Liu and Cui et al., 2018; Huang and Ding et al., 2020). Several potential mechanisms can be used to explain the harm of $\mathrm{PM}_{2.5}$ on TB: (1) When $\mathrm{PM}_{2.5}$ exposure is increased, the immune function of human respiratory system will be altered or impaired. Exposure may harm lung immunity by inducing nitrosation stressors and oxidation (Nel, 2005). (2) $\mathrm{PM}_{2.5}$ can weaken mucociliary clearance function and alveolar macrophage activity (D'Amato and Cecchi et al., 2010; Smith and Paszkiewicz et al., 2010). (3) $\mathrm{PM}_{2.5}$ affects the immune function of anti-mycobacterium $\mathrm{T}$ cells by disrupting the synthesis and secretion of inflammatory factors (Ibironke and Carranza et al., 2019). (4) $\mathrm{PM}_{2.5}$ can carry minerals, metal ions and other external impurities into the bronchioles and alveoli, causing various forms of inflammation and damaging the body's immune function (Bai and Su et al., 2018; Cheng and Zhu et al., 2019; Popovic and Soares et al., 2019). Therefore, in combination with the results of this study and previous studies, it can be seen that $\mathrm{PM}_{2.5}$ can damage the immune response of the lungs and accelerate the progress of tuberculosis. We can see that from some epidemiological studies, $\mathrm{PM}_{10}$, it has a similar effect and reaction mechanism to $\mathrm{PM}_{2.5}$ (Rivas-Santiago and Sarkar et al., 2015; Kim and Yu et al., 2020; Pompilio and Di Bonaventura, 2020; 
Xiang and $\mathrm{Xu}$ et al., 2021). $\mathrm{PM}_{2.5}$ and $\mathrm{PM}_{10}$ are so small in diameter that they can pass through the physical barrier of the respiratory tract. The aerosol nuclei containing Mycobacterium tuberculosis were colonized deep in lung tissue with $\mathrm{PM}_{2.5}$ and $\mathrm{PM}_{10}$ as carriers. This is also a risk factor for TB infection (Yang and Zhang et al., 2020).

We observed a protective effect of sulfur dioxide. Similar protective effects have been reported in cities such as Madrid in Spain and Ningbo and Hefei in China (AlvaroMeca and Diaz et al., 2016; Ge and Fan et al., 2017; Huang and Ding et al., 2020). This protective effect of $\mathrm{SO}_{2}$ is thought to be due to the antibacterial properties of $\mathrm{SO}_{2}$. The ability of $\mathrm{SO}_{2}$ to block enzyme activity in microbial cell membranes may account for the antimicrobial properties (Ge and Fan et al., 2017). An in vitro study of $\mathrm{SO}_{2}$ also demonstrated its inhibitory effect on the growth of Mycobacterium tuberculosis (Malwal and Sriram et al., 2011). One study reported that male mice inhaled small amounts of $\mathrm{SO}_{2}$ can increased levels of inflammatory cytokines in lung tissue and inhibited the proliferation of Mycobacterium tuberculosis (Meng and Liu et al., 2005). However, some related studies have obtained the opposite result of our conclusion, that $\mathrm{SO}_{2}$ is a risk factor (Liu and Zhang et al., 2020; Liu and Zhao et al., 2021). Therefore, it is necessary to further study the mechanism of action of $\mathrm{SO}_{2}$ in the human body to provide a more comprehensive and scientific explanation.

With regard to $\mathrm{O}_{3}$, we observed a positive correlation with the risk of TB, the lag effect was observed to be statistically significant at 0 day, indicating an acute effect of $\mathrm{O}_{3}$ on the human body. The independent effect of $\mathrm{O}_{3}$ on increased TB risk also was observed in a multi-city model study in Shandong Province, China (Liu and Zhao et al., 
2021). Laboratory evidence has shown that $\mathrm{O}_{3}$ increases the incidence of airway inflammation, impairs lung function and affects lung gas exchange (Smith and Van Den Eeden et al., 2016). However, a nested case-control study in Northern California showed that $\mathrm{O}_{3}$ exposure above the lowest quintile resulted in a reduced risk of tuberculosis. A time series study from Hefei, China, also concluded that $\mathrm{O}_{3}$ reduces the risk of tuberculosis outpatient visits (Smith and Van Den Eeden et al., 2016; Huang and Ding et al., 2020). Due to the differences in study results and the unclear biological mechanisms of $\mathrm{O}_{3}$ associated with TB infection, further studies are recommended to explain the effect of $\mathrm{O}_{3}$ on TB risk.

The results of this study suggested that $\mathrm{CO}$ exposure increases the risk of TB outpatient visits. Similar protective effects have been reported in northern California, those exposed to $\mathrm{CO}$ in the highest quintile had a $50 \%$ increase in $\mathrm{TB}$ prevalence compared with those exposed to the lowest quintile (Smith and Van Den Eeden et al., 2016). Two studies in Wuhan and Jinan in China showing positive effects of CO on TB (Liu and Cui et al., 2018; Xu and Liao et al., 2019). One study suggests that heme oxygenase is produced by carbon monoxide, which inhibits the body's immune response and inflammation (Chin and Otterbein, 2009). In addition, CO may activate the interleukin-10 cascade in pulmonary macrophages, leading to $M$. tuberculosis reactivation. Microbiological studies have shown that Mycobacterium can use $\mathrm{CO}$ as carbon source and energy source for growth and reproduction under aerobic conditions (Park and Hwang et al., 2003; Tremblay, 2007). An experiment in mice found that exposure to diesel exhaust, including $\mathrm{CO}$, reduced mRNA expression of several pro- 
inflammatory cytokines and cell-signaling enzymes that control Mycobacterium tuberculosis infection (Hiramatsu and Saito et al., 2005).

Our study showed a positive correlation between $\mathrm{NO}_{2}$ and $\mathrm{TB}$, two time series studies in Chengdu and Hefei also reached similar conclusions (Zhu and Xia et al., 2018; Huang and Ding et al., 2020). A study in Taiwan has shown that $\mathrm{NO}_{2}$ is associated with tuberculosis which contributed 1.2-9.8\% to developing TB (Lin and Lin et al., 2019). Regarding the mechanism of action of $\mathrm{NO}_{2}$, related studies have shown that inhalation of $\mathrm{NO}_{2}$ may reduce the ability of mice to kill bacteria in the lungs and increase the risk of respiratory bacterial infection (Goldstein and Eagle et al., 1973). From a biochemical point of view, $\mathrm{NO}_{2}$ is an irritating gas that can corrode and damage the alveoli and lower respiratory tract, and $\mathrm{NO}_{2}$ is a component of photochemical smog and has acute toxic effects on the lungs (Wang and Chen et al., 2014; Smith and Van Den Eeden et al., 2016). These may be the reasons for $\mathrm{NO}_{2}$ 's influence on TB outpatient visit risk.

In the subgroup analysis, we found that pollutant exposure had a more significant impact on the male group, and all pollutants except $\mathrm{NO}_{2}$ were statistically significant. This may be due to men's greater exposure to outdoor environments and lifestyle factors such as smoking and drinking. In addition, there are significant differences in hormonal status and airway physiology between males and females, which may also be one of the reasons (Sopori and Kozak et al., 1998; Przybylski and Nowakowska-Arendt et al., 2014). In the age subgroup, the effects of pollutants on older age groups were greater. The possible reason is that with age, the body's immune defenses decline, and the body is more likely to be exposed to pollutants than younger people (Zhang and Ni et al., 
2019; Huang and Ding et al., 2020). Interestingly, contrary results were observed for $\mathrm{PM}_{2.5}$ and $\mathrm{PM}_{10}$ in the cold and warm seasons. In the cold season group, the risk of tuberculosis diagnosis was still increased, while in the warm season group, $\mathrm{PM}_{2.5}$ and $\mathrm{PM}_{10}$ reached statistical significance when they lagged by 2 days and 1 day, respectively, reducing the risk of tuberculosis outpatient visits. Some possible reasons can explain this phenomenon:(1) The temporal distribution of particulate matter pollutants showed obvious seasonal differences, with high in cold season and low in warm season. (2) During periods of high ambient air pollutants, particularly particulate matter exposure at high concentrations visible to the naked eye, humans prefer to stay indoors, leading to increased exposure and further increasing the risk of tuberculosis (Huang and Xiang et al., 2020). But the actual mechanism remains unclear, and more research is needed to determine the cause.

Our study has several strengths. First, we explored for the first time the relationship between air pollution exposure and tuberculosis outpatient visits in Fuyang city, which is the most populous city in Anhui province and has a good representation. Second, the impact of six contaminants on TB visit risk was thoroughly explored, and the sensitivity analysis showed that the results were robust and reliable. However, there are still some shortcomings. First of all, this study adopted the average daily concentration of fixed stations as the exposure concentration of air pollution, without excluding the influence of spatial heterogeneity of air pollution. Second, the ecological fallacy is inevitable. Third, this study was limited to one area in Fuyang, which means that our findings should be carefully extended to other areas. 


\section{Conclusion}

outpatient visits. $\mathrm{PM}_{10}, \mathrm{PM}_{2.5}, \mathrm{NO}_{2}, \mathrm{O}_{3}$ and $\mathrm{CO}$ increased the number of tuberculosis

outpatient visits, while $\mathrm{SO}_{2}$ decreased the number of tuberculosis outpatient visits. Men

and the elderly are more likely to be affected by air pollution, and the effects of season

are more pronounced than age or sex. Therefore, we should give more comprehensive prevention and treatment to high-risk groups. Meanwhile add more measures to against the effects of seasonal changes. and Ying-Qing Li analyzed the data and drafted the manuscript, Xiao-Hong Kan and

Acknowledgements We would like to thank all the subjects who participated in this research. This work was supported by Anhui Medical University (2019xkj019), Anhui Provincial Natural Science Foundation (2008085MH063), National Key Project for Infectious Disease (2018ZX10722301-001-004) and Major national science and technology projects during the 12th Five-Year Plan period (2013ZX10003008-001$003)$.

Author's contributions Xin-Qiang Wang and Xiao-Hong Kan collaboratively designed the study, both making substantial intellectual contribution. Xin-Qiang Wang Xiu-Jun Zhang revised the manuscript. Cheng-Yang Hu, Kai Huang, Kun Ding, Xiao- 
Jing Yang, Xin Cheng, Kang-Di Zhang, Wen-Jie Yu, Jie Wang, Yong-Zhong Zhang, Zhen-tao Ding contributed $\mathrm{n}$ collecting the data. Xin-Qiang Wang and Ying-Qing Li contributed equally. All authors read and approved the final manuscript.

Funding This study was supported by Anhui Medical University (2019xkj019), Anhui Provincial Natural Science Foundation (2008085MH063), National Key Project for Infectious Disease (2018ZX10722301-001-004) and Major national science and technology projects during the 12th Five-Year Plan period (2013ZX10003008-001$003)$.

Data availability The datasets analyzed during the current study are available from the corresponding author on reasonable request.

\section{Declarations}

Ethical approval Not applicable.

Consent to participate Not applicable.

Consent for publication Not applicable.

Competing interests The authors declare no competing interests.

\section{References:}

Alvaro-Meca, A. and A. Diaz, et al. (2016). "Environmental Factors Related to 
Pulmonary Tuberculosis in HIV-Infected Patients in the Combined Antiretroviral Therapy (cART) Era." PLoS One 11 (11): e0165944. https://doi.org/10.1371/journal.pone.0165944.

Bhaskaran, K. and A. Gasparrini, et al. (2013). "Time series regression studies in environmental epidemiology." Int $\mathrm{J} \quad$ Epidemiol $\mathbf{4 2}$ (4): 1187-95. https://doi.org/10.1093/ije/dyt092.

Chen, F. and Z. Deng, et al. (2017). "Attributable risk of ambient PM10 on daily mortality and years of life lost in Chengdu, China." Science of The Total Environment 581-582: 426-433. https://doi.org/10.1016/j.scitotenv.2016.12.151.

Chen, R. and C. Chu, et al. (2010). "Ambient air pollution and hospital admission in Shanghai, China." J Hazard Mater 181 (1-3): 234-40. https://doi.org/10.1016/j.jhazmat.2010.05.002.

Chin, B. Y. and L. E. Otterbein (2009). "Carbon monoxide is a poison... to microbes! $\mathrm{CO}$ as a bactericidal molecule." Curr Opin Pharmacol 9 (4): 490-500. https://doi.org/10.1016/j.coph.2009.06.025.

D'Amato, G. and L. Cecchi, et al. (2010). "Urban air pollution and climate change as environmental risk factors of respiratory allergy: an update." J Investig Allergol Clin Immunol 20 (2): 95-102; quiz following 102.

Elf, J. L. and O. Eke, et al. (2017). "Indoor air pollution from secondhand tobacco smoke, solid fuels, and kerosene in homes with active tuberculosis disease in South Africa." BMC Research Notes 10 (1). https://doi.org/10.1186/s13104-017$2892-2$. 
Gasparrini, A. (2014). "Modeling exposure-lag-response associations with distributed lag non-linear models." Stat Med 33 (5): 881-99. https://doi.org/10.1002/sim.5963.

Gasparrini, A. and B. Armstrong, et al. (2010). "Distributed lag non-linear models." Stat Med 29 (21): 2224-34. https://doi.org/10.1002/sim.3940.

Ge, E. and M. Fan, et al. (2017). "Ambient sulfur dioxide levels associated with reduced risk of initial outpatient visits for tuberculosis: A population based time series analysis." $\quad$ Environmental Pollution 228: 408-415. https://doi.org/10.1016/j.envpol.2017.05.051.

Goldstein, E. and M. C. Eagle, et al. (1973). "Effect of nitrogen dioxide on pulmonary bacterial defense mechanisms." Arch Environ Health 26 (4): 202-4. https://doi.org/10.1080/00039896.1973.10666256

Guo, Y. and Y. Ma, et al. (2017). "Time series analysis of ambient air pollution effects on daily mortality." Environ Sci Pollut Res Int 24 (25): 20261-20272. https://doi.org/10.1007/s11356-017-9502-7.

Hiramatsu, K. and Y. Saito, et al. (2005). "The effects of inhalation of diesel exhaust on murine mycobacterial infection." Exp Lung Res 31 (4): 405-15. https://doi.org/10.1080/01902140590918786.

Huang, K. and K. Ding, et al. (2020). "Association between short-term exposure to ambient air pollutants and the risk of tuberculosis outpatient visits: A time-series study in Hefei, China." Environmental Research 184: 109343. https://doi.org/10.1016/j.envres.2020.109343.

Huang, K. and X. Yang, et al. (2020). "Short-term effect of ambient temperature change 
on the risk of tuberculosis admissions: Assessments of two exposure metrics."

189:

109900. https://doi.org/10.1016/j.envres.2020.109900.

Huang, S. and H. Xiang, et al. (2020). "Short-Term Effect of Air Pollution on Tuberculosis Based on Kriged Data: A Time-Series Analysis." International Journal of Environmental Research and Public Health 17 (5): 1522. https://doi.org/10.3390/ijerph17051522.

Hwang, S. and S. Kang, et al. (2014). "Impact of outdoor air pollution on the incidence of tuberculosis in the Seoul metropolitan area, South Korea." The Korean Journal of Internal Medicine 29 (2): 183. https://doi.org/10.3904/kjim.2014.29.2.183.

Jassal, M. S. and I. Bakman, et al. (2013). "Correlation of ambient pollution levels and heavily-trafficked roadway proximity on the prevalence of smear-positive $\begin{array}{lllll}\text { tuberculosis." } & \text { Public } & \text { Health } & 127 & \text { (3): }\end{array}$ https://doi.org/10.1016/j.puhe.2012.12.030.

Kim, H. and S. Yu, et al. (2020). "Effects of particulate air pollution on tuberculosis development in seven major cities of Korea from 2010 to 2016: methodological considerations involving long-term exposure and time lag." Epidemiology and Health 42: e2020012. https://doi.org/10.4178/epih.e2020012.

Kim, J. (2014). "Is ambient air pollution another risk factor of tuberculosis?" Korean J Intern Med 29 (2): 170-2. https://doi.org/10.3904/kjim.2014.29.2.170.

Li, Z. and X. Mao, et al. (2019). "Long-term effect of exposure to ambient air pollution on the risk of active tuberculosis." Int $\mathrm{J}$ Infect Dis 87: 177-184. 
Lin, Y. and H. Lin, et al. (2019). " $<\mathrm{p}>$ Association Between Ambient Air Pollution and Elevated Risk of Tuberculosis Development</p>." Volume 12: 3835-3847. https://doi.org/10.2147/IDR.S227823.

Liu, F. and Z. Zhang, et al. (2020). "Associations of ambient air pollutants with regional pulmonary tuberculosis incidence in the central Chinese province of Hubei: a Bayesian spatial-temporal analysis." Environmental Health 19 (1). https://doi.org/10.1186/s12940-020-00604-y.

Liu, H. and Y. Tian, et al. (2017). "Association between ambient air pollution and hospitalization for ischemic and hemorrhagic stroke in China: A multicity case$\begin{array}{lllll}\text { crossover } & \text { study." } & \text { Environ } & \text { Pollut 230: } & \text { 234-241. }\end{array}$ https://doi.org/10.1016/j.envpol.2017.06.057.

Liu, Y. and L. Cui, et al. (2018). "Ambient Air Pollution Exposures and Newly Diagnosed Pulmonary Tuberculosis in Jinan, China: A Time Series Study." Scientific Reports 8 (1). https://doi.org/10.1038/s41598-018-35411-6.

Liu, Y. and S. Zhao, et al. (2021). "Effect of ambient air pollution on tuberculosis risks and mortality in Shandong, China: a multi-city modeling study of the short- and long-term effects of pollutants." Environmental Science and Pollution Research. https://doi.org/10.1007/s11356-021-12621-6.

Malwal, S. R. and D. Sriram, et al. (2011). "Design, Synthesis, and Evaluation of ThiolActivated Sources of Sulfur Dioxide (SO2) as Antimycobacterial Agents." Journal of Medicinal Chemistry 55 (1): 553-557. https://doi.org/10.1021/jm201023g. 
Meng, Z. and Y. Liu, et al. (2005). "Effect of sulfur dioxide inhalation on cytokine levels in lungs and serum of mice." Inhal Toxicol 17 (6): 303-7. https://doi.org/10.1080/08958370590922625.

Nel, A. (2005). "Atmosphere. Air pollution-related illness: effects of particles." Science 308 (5723): 804-6. https://doi.org/10.1126/science.1108752.

Park, S. W. and E. H. Hwang, et al. (2003). "Growth of Mycobacteria on Carbon Monoxide and Methanol." Journal of Bacteriology 185 (1): 142-147. https://doi.org/10.1128/jb.185.1.142-147.2003.

Pompilio, A. and G. Di Bonaventura (2020). "Ambient air pollution and respiratory bacterial infections, a troubling association: epidemiology, underlying mechanisms, and future challenges." Critical reviews in microbiology 46 (5): 600630. https://doi.org/10.1080/1040841x.2020.1816894.

Popovic, I. and R. J. Soares Magalhaes, et al. (2019). "A systematic literature review and critical appraisal of epidemiological studies on outdoor air pollution and tuberculosis outcomes." Environmental Research 170: 33-45. https://doi.org/10.1016/j.envres.2018.12.011.

Przybylski, G. and A. Nowakowska-Arendt, et al. (2014). "[10 years comparative clinico-epidemiological analysis of smoking and alcohol consumption in TB patients (Myc. Tuberculosis) and with mycobacteriosis (Myc. Kansas]." Przegl Lek 71 (11): 576-80.

Rivas-Santiago, C. E. and S. Sarkar, et al. (2015). "Air Pollution Particulate Matter Alters Antimycobacterial Respiratory Epithelium Innate Immunity." Infection and 
Sarkar, S. and C. E. Rivas-Santiago, et al. (2019). "Season and size of urban particulate matter differentially affect cytotoxicity and human immune responses to Mycobacterium tuberculosis." PLOS ONE $14 \quad$ (7): 00219122. https://doi.org/10.1371/journal.pone.0219122.

Smith, G. S. and S. K. Van Den Eeden, et al. (2016). "Air Pollution and Pulmonary Tuberculosis: A Nested Case-Control Study among Members of a Northern California Health Plan." Environ Health Perspect 124 (6): 761-8. https://doi.org/10.1289/ehp.1408166.

Smith, G. S. and V. J. Schoenbach, et al. (2014). "Particulate air pollution and susceptibility to the development of pulmonary tuberculosis disease in North Carolina: an ecological study." Int J Environ Health Res 24 (2): 103-12. https://doi.org/10.1080/09603123.2013.800959.

Smith, L. A. and G. M. Paszkiewicz, et al. (2010). "Inflammatory response of lung macrophages and epithelial cells to tobacco smoke: a literature review of ex vivo investigations." Immunol Res 46 (1-3): 94-126. https://doi.org/10.1007/s12026009-8133-6.

Sopori, M. L. and W. Kozak, et al. (1998). "Nicotine-induced modulation of T Cell function. Implications for inflammation and infection." Adv Exp Med Biol 437: 279-89. https://doi.org/10.1007/978-1-4615-5347-2_31.

Torres, M. and C. Carranza, et al. (2019). "Urban airborne particle exposure impairs human lung and bloodMycobacterium tuberculosis immunity." Thorax 74 (7): 
Tremblay, G. A. (2007). "Historical statistics support a hypothesis linking tuberculosis and air pollution caused by coal." Int J Tuberc Lung Dis 11 (7): 722-32.

Wang, W. and W. Guo, et al. (2021). "Epidemiological characteristics of tuberculosis and effects of meteorological factors and air pollutants on tuberculosis in Shijiazhuang, China: A distribution lag non-linear analysis." Environmental Research 195: 110310. https://doi.org/10.1016/j.envres.2020.110310.

Wang, X. and J. Chen, et al. (2014). "Particle number concentration, size distribution and chemical composition during haze and photochemical smog episodes in

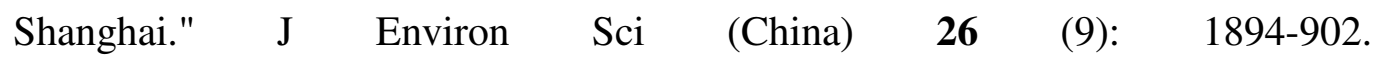
https://doi.org/10.1016/j.jes.2014.07.003.

WHO (2020). "Global tuberculosis report 2020.".

Xiang, K. and Z. Xu, et al. (2021). "Association between ambient air pollution and tuberculosis risk: A systematic review and meta-analysis." Chemosphere 277: 130342. https://doi.org/10.1016/j.chemosphere.2021.130342.

Xu, M. and J. Liao, et al. (2019). "Association of air pollution with the risk of initial outpatient visits for tuberculosis in Wuhan, China." Occupational and Environmental Medicine 76 (8): 560-566. https://doi.org/10.1136/oemed-2018105532.

Yang, J. and M. Zhang, et al. (2020). "A study on the relationship between air pollution and pulmonary tuberculosis based on the general additive model in Wulumuqi, China." International Journal of Infectious Diseases 96: 42-47. 
https://doi.org/10.1016/j.ijid.2020.03.032.

Zhang, Y. and H. Ni, et al. (2019). "The short-term association between air pollution and childhood asthma hospital admissions in urban areas of Hefei City in China: A time-series study." Environ Res 169: 510-516. https://doi.org/10.1016/j.envres.2018.11.043.

Zhu, S. and L. Xia, et al. (2018). "Ambient air pollutants are associated with newly diagnosed tuberculosis: A time-series study in Chengdu, China." Science of The Total Environment 631-632: 47-55. https://doi.org/10.1016/j.scitotenv.2018.03.017.

Figure Captions:

Fig. 1. The geographical location of Fuyang City and tuberculosis outpatient number in cities of Anhui Province from 2015-2020.

Fig. 2. Spearman rank correlation coefficients and scatter plot between daily air pollutant concentrations and meteorological factors in Fuyang, 2015-2020. *: $\mathrm{r}_{\mathrm{s}}>0.7$

Fig. 3. Time series of Tuberculosis, O3, PM2.5, PM10, SO2, NO2, and CO in Fuyang, China, from 2015 to 2020.

Fig .4. Lag-specific relative risks (95\% CI) and cumulative risks (95\% CI) of per 10unit increase in the daily concentrations of air pollution on TB outpatient visits on different lag days, according to the single-pollutant model; TB, tuberculosis; PM2.5, particulate matter $<2.5 \mu \mathrm{m}$ in aerodynamic diameter; PM10, particulate matter $<10$ $\mu \mathrm{m}$ in aerodynamic diameter; $\mathrm{CO}$, carbon monoxide; $\mathrm{O} 3$, ozone; $\mathrm{SO}$, sulfur dioxide; 

NO2, nitrogen dioxide.

Fig .5. Lag-specific relative risks $(95 \% \mathrm{CI})$ of per 10-unit increase in the daily 663 concentrations of air pollution on TB outpatient visits on different lag days, according 664 to the single-pollutant model stratified by age; TB, tuberculosis; PM2.5, particulate 665 matter $<2.5 \mu \mathrm{m}$ in aerodynamic diameter; PM10, particulate matter $<10 \mu \mathrm{m}$ in aerodynamic diameter; $\mathrm{CO}$, carbon monoxide; O3, ozone; SO2, sulfur dioxide; NO2, 667 nitrogen dioxide.

Fig .6. Lag-specific relative risks $(95 \% \mathrm{CI})$ of per 10-unit increase in the daily concentrations of air pollution on TB outpatient visits on different lag days, according to the single-pollutant model stratified by gender and season; TB, tuberculosis; PM2.5, 671 particulate matter $<2.5 \mu \mathrm{m}$ in aerodynamic diameter; PM10, particulate matter $<10$ $672 \mu \mathrm{m}$ in aerodynamic diameter; $\mathrm{CO}$, carbon monoxide; O3, ozone; SO2, sulfur dioxide; 673 NO2, nitrogen dioxide. 
Figures

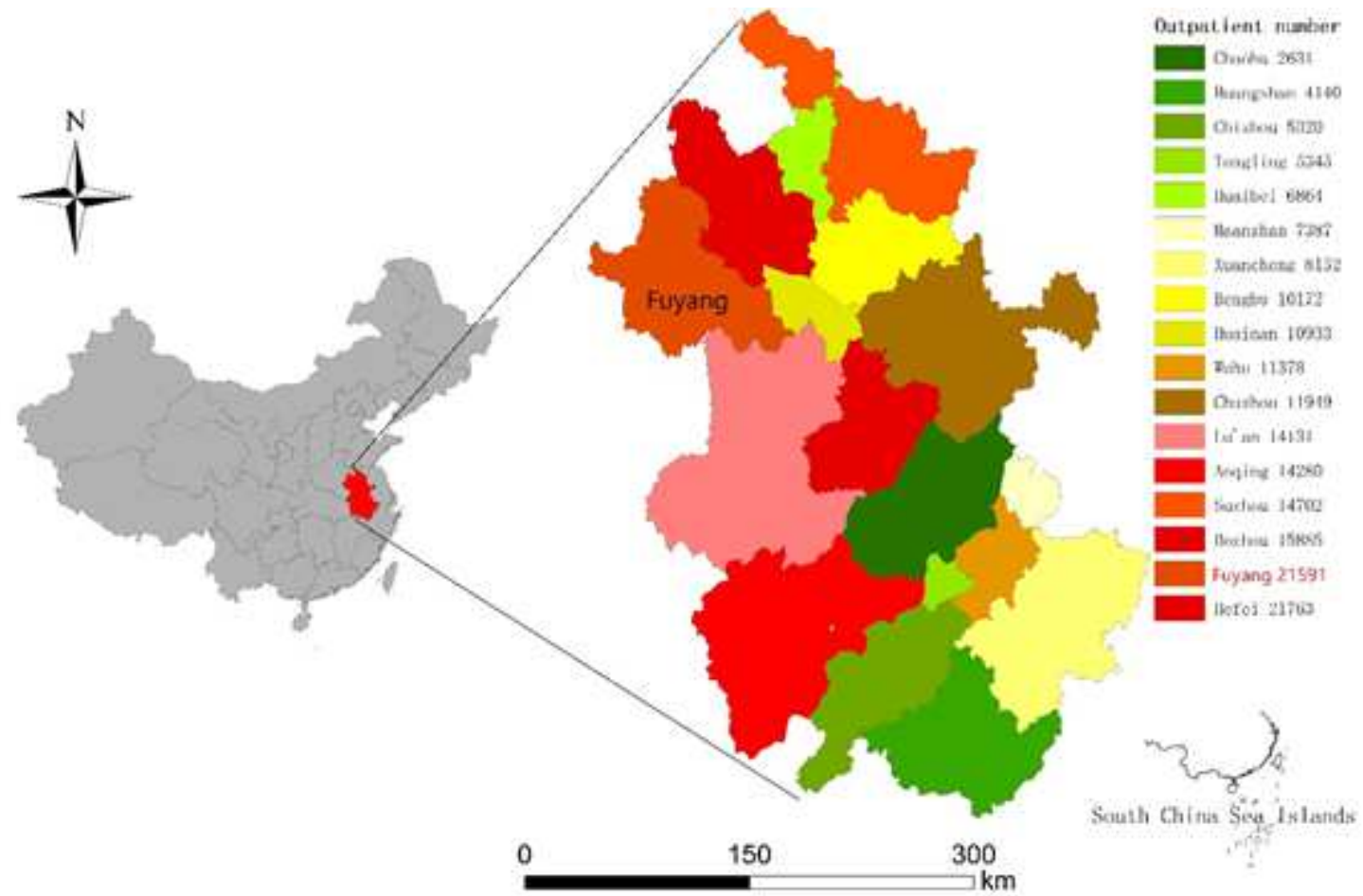

Figure 1

The geographical location of Fuyang City and tuberculosis outpatient number in cities of Anhui Province from 2015-2020. 


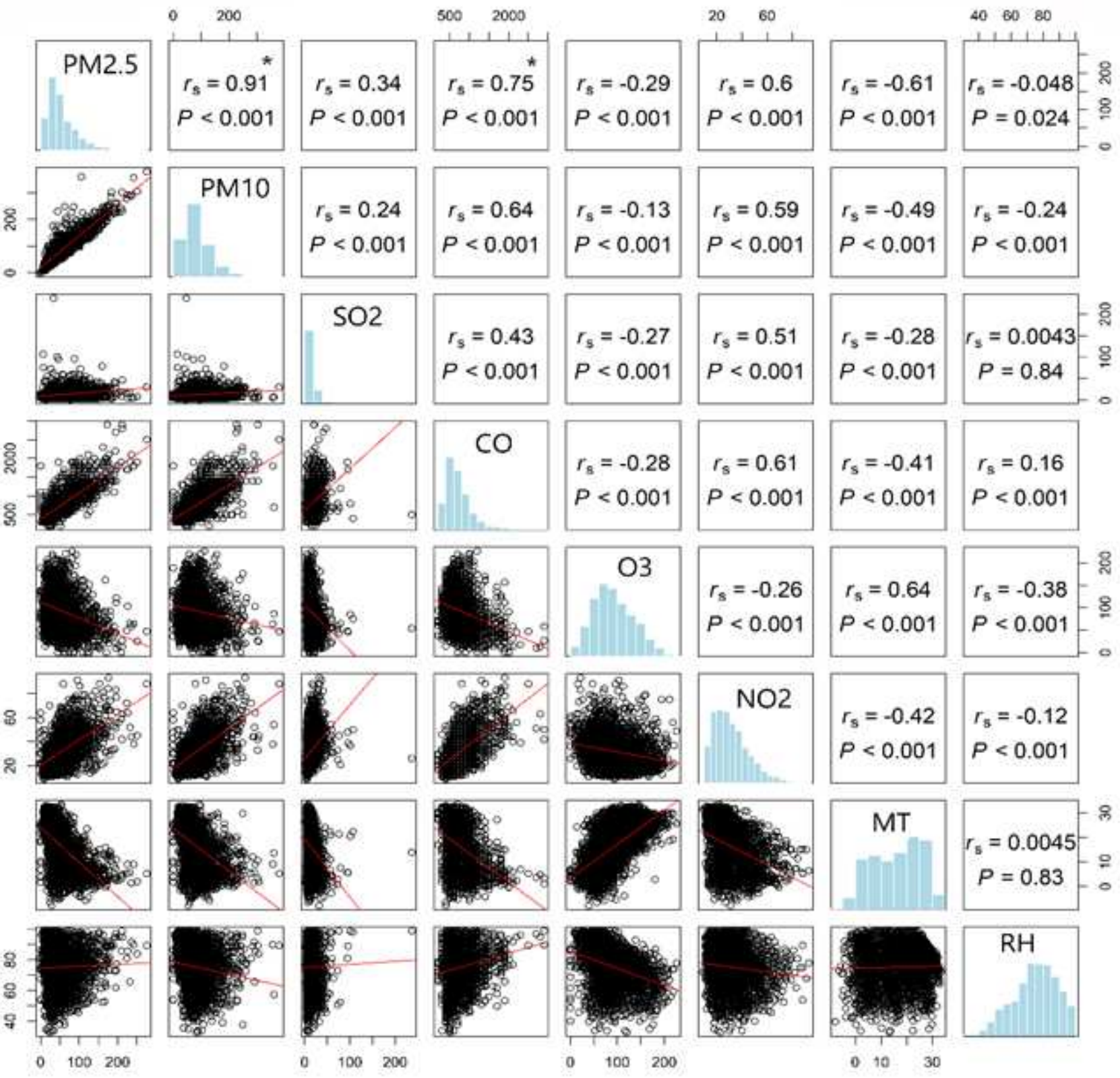

Figure 2

Spearman rank correlation coefficients and scatter plot between daily air pollutant concentrations and meteorological factors in Fuyang, 2015-2020. *: rs > 0.7 . 

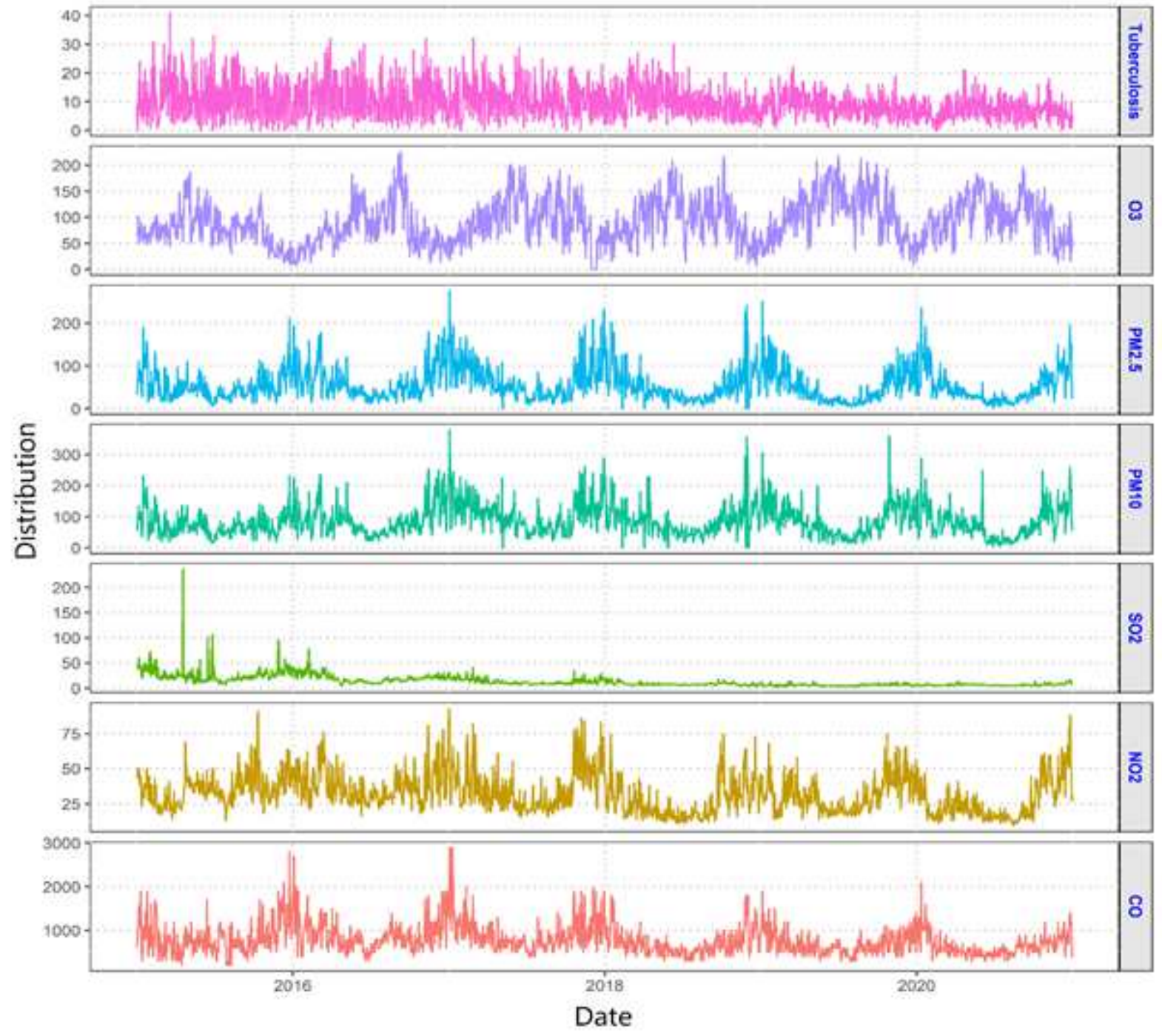

Figure 3

Time series of Tuberculosis, O3, PM2.5, PM10, SO2, NO2, and CO in Fuyang, China, from 2015 to 2020. 

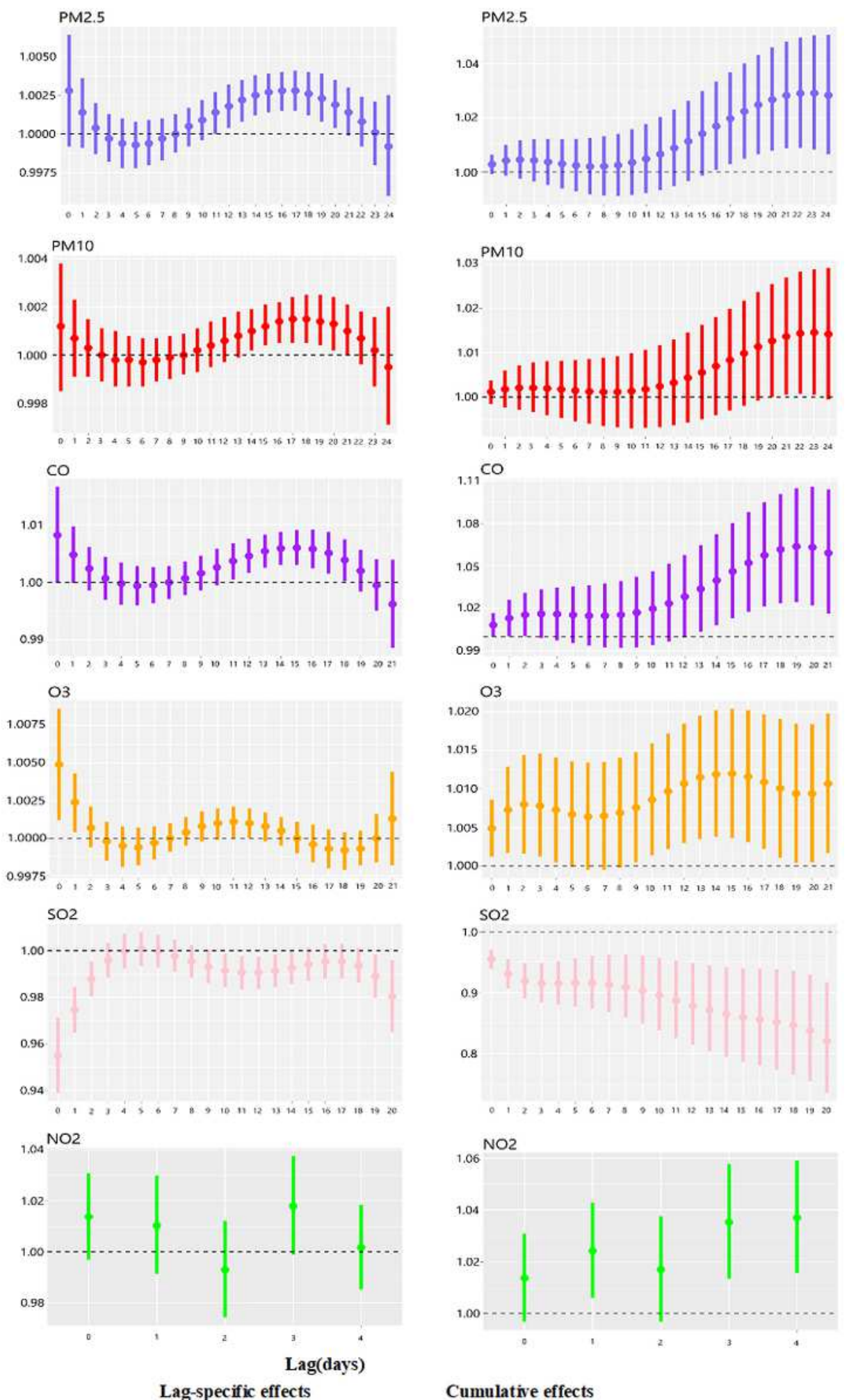

Figure 4

Lag-specific relative risks $(95 \% \mathrm{Cl})$ and cumulative risks $(95 \% \mathrm{Cl})$ of per 10 -unit increase in the daily concentrations of air pollution on TB outpatient visits on different lag days, according to the singlepollutant model; TB, tuberculosis; PM2.5, particulate matter < $2.5 \mu \mathrm{m}$ in aerodynamic diameter; PM10, particulate matter < $10 \mu \mathrm{m}$ in aerodynamic diameter; $\mathrm{CO}$, carbon monoxide; 03, ozone; SO2, sulfur dioxide; NO2, nitrogen dioxide. 


\section{Age $<15$ years $\phi \geq 65$ years $\$ 15-65$ years}
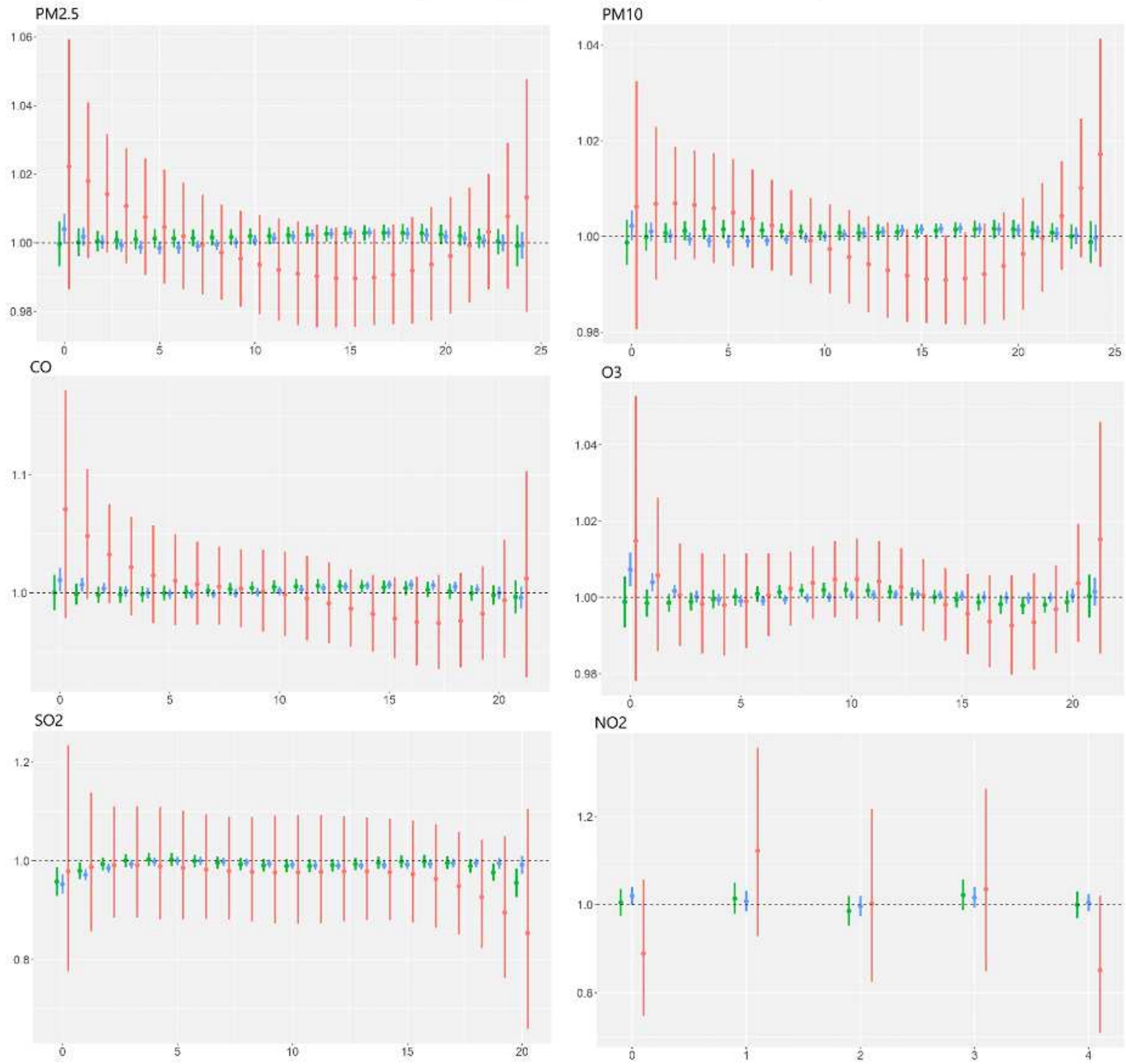

Lag(days)

\section{Figure 5}

Lag-specific relative risks $(95 \% \mathrm{Cl})$ of per 10 -unit increase in the daily concentrations of air pollution on TB outpatient visits on different lag days, according to the single-pollutant model stratified by age; TB, tuberculosis; PM2.5, particulate matter < $2.5 \mu \mathrm{m}$ in aerodynamic diameter; PM10, particulate matter < 10 $\mu \mathrm{m}$ in aerodynamic diameter; $\mathrm{CO}$, carbon monoxide; $\mathrm{O} 3$, ozone; $\mathrm{SO} 2$, sulfur dioxide; $\mathrm{NO} 2$, nitrogen dioxide 

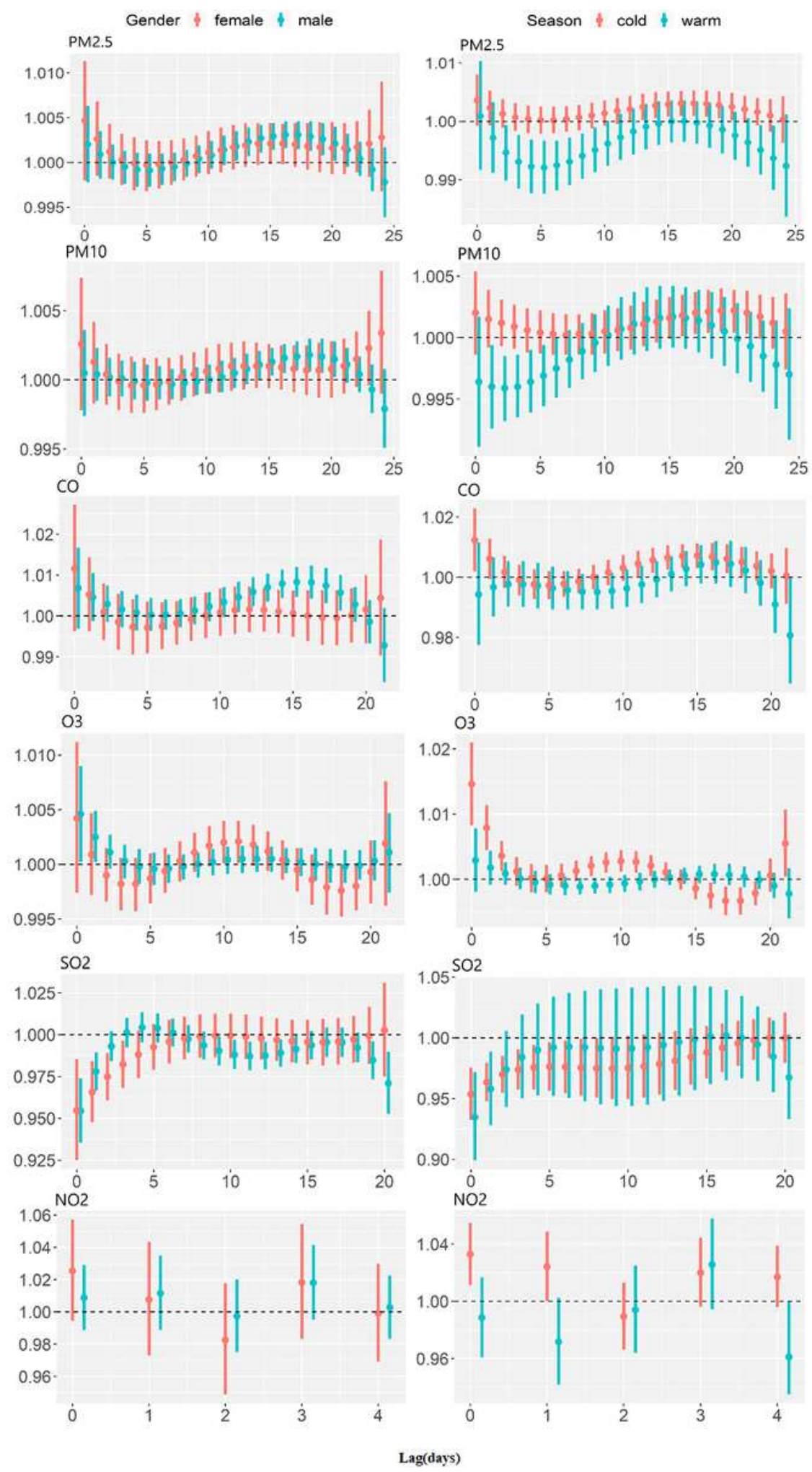

Figure 6

Lag-specific relative risks $(95 \% \mathrm{Cl})$ of per 10 -unit increase in the daily concentrations of air pollution on TB outpatient visits on different lag days, according to the single-pollutant model stratified by gender and season; TB, tuberculosis; PM2.5, particulate matter < $2.5 \mu \mathrm{m}$ in aerodynamic diameter; PM10, particulate matter < $10 \mu \mathrm{m}$ in aerodynamic diameter; $\mathrm{CO}$, carbon monoxide; 03, ozone; SO2, sulfur dioxide; NO2, nitrogen dioxide. 


\section{Supplementary Files}

This is a list of supplementary files associated with this preprint. Click to download.

- SupplementaryFigure.docx

- SupplementaryTable.docx 